\section{Evaluation of Alvarado Score in Diagnosing Acute Appendicitis}

\section{Abstract}

Background: Using a reliable scoring system to diagnose acute appendicitis can help reduce the rate of unnecessary surgery and, henceforth, its complications. This cohort study aimed to evaluate Alvarado scoring system for diagnosing acute appendicitis in our health facility.

Methods: 100 patients of different age groups with clinical suspicion of acute appendicitis admitted in the General Surgical Ward, Karak Teaching Hospital in Karak, Jordan, from April, 2013 to December, 2014 were included in the study. All patients underwent surgery and were grouped according to the variables of Alvarado scoring system and then divided into two groups. Group I patients (score 7 or more), group II patients (scores 3-5, and 5-6). Diagnosis was confirmed by histopathologic examination. Reliability of scoring system was assessed by calculating negative appendectomy rate and positive predictive value.

Results: Out of total 100 patients, appendicitis was confirmed 80 patients, thus giving negative appendectomy rate of $20 \%$ (male $6 \%$, female $16 \%$ ). Perforation rate was $4 \%$, positive predictive value was $90 \%$, negative predictive value of $29 \%$, sensitivity of $54 \%$, specificity $75 \%$, and diagnostic accuracy value of $58 \%$.

Conclusion: Alvarado score is not sensitive tool for aiding diagnosis of acute appendicitis.

Keywords: Acute appendicitis; Appendectomy; Scoring system; Alvarado Score

\section{Awayshih MMA*, Yousef AJ and Nofal MN}

\author{
Department of General Surgery and \\ Anesthesia, College of Medicine, Mutah \\ University, Jordan
}

*Corresponding author:

Awayshih MMA

”dr.mahmoud60@yahoo.com

Department of General Surgery and Anesthesia, College of Medicine, Mutah University, Jordan.

Tel: +00962790662682

Citation: Awayshih MMA, Yousef AJ, Nofal MN (2019) Evaluation of Alvarado Score in Diagnosing Acute Appendicitis. J Univer Surg Vol.7 No.1:3

Received: May 09, 2019; Accepted: May 27, 2019; Published: June 03, 2019

\section{Introduction}

Acute appendicitis is the most common abdominal surgical emergency with a lifetime cumulative incidence of $7 \%$ [1]. In 1886; Fitz described the traditional signs and symptoms of acute apendicitis [2].

The diagnosis of acute appendicitis is basically clinical depending on history, clinical examination and sometimes aided by laboratory investigations (such as white blood cells count or CRP level). Imaging modalities are not requested routinely because they have been shown to add very little information unless there are complications. The definitive diagnosis is achieved at surgery and after histopathologic examination of the resected appendix [3].

Delay in diagnosis and management may result in significant morbidity and, sometimes, mortality. A number of scoring systems have been developed for aiding early diagnosis of acute appendicitis. Previous reports mentioned that scoring systems are a valuable and valid instrument of discrimination between acute appendicitis and nonspecific abdominal pain [4].

Alvarado scoring system, introduced in 1986, is one of these systems and is based on history, clinical examination and few laboratory findings (Table 1) [5].

\section{Materials and Methods}

This study was carried out on 100 consecutive patients admitted to the Surgical Ward of Karak Teaching Hospital, Karak-Jordan, with the clinical diagnosis of acute appendicitis during the period from April 2013 to December 2014. Patients of any age group and both sexes presenting to the emergency department with pain in right iliac fossa pain and operated with appendectomy were included in the study. Patients with clear presentation of other diagnoses such as urological, gynaecological or acute abdomen conditions other than appendicitis were excluded from the study.

All included patients were admitted after initial assessment in the emergency department and base-line investigations 
including complete blood count, urine routine examination, and serum bilirubin level. Then study format was filled in for each patient by a general surgery officer. The format consisted of general patient demographics and Alvarado score variables. The sums of all the scores were calculated for each patient and according to the results patients were divided into two groups: Group I Alvarado score $<7$ (low and intermediate suspicion group), and group II Alvarado score $\geq 7$ (high suspicion group). All the patients underwent appendectomy after variable hours of observation, and the surgical specimens were examined grossly and pathologically. The formats were completed with pathology reports and post operative course.

Finally the sensitivity, specificity, positive predictive value, negative predictive value, and diagnostic accuracy value of Alvarado scoring system were calculated.

\section{Results}

Our study was conducted on one hundred consecutive patients with clinical picture of acute appendicitis. Among these patients 44 were female $(44 \%)$ and 56 were male $(56 \%)$. The male to female ratio was 1:1.2. Mean age was 22.9 years (range $5-61$ years, standard deviation +12.5 years), with median age of 19 years. The frequency distribution of patients according to Alvarado scoring system is shown in Table 2.

In group I we had 52 patients totally: 2 patients (2\%) with Alvarado score of 3-5, one female and one male, and both had unremarkable appendix pathology. 50 patients (50\%) with Alvarado score of 5-7, 25 males and 25 females: The appendix was perforated in two patients, a 40-year old female and a 8-year old male; gangrenous in one patient: A 13-year old female, and unremarkable in 13 patients: 10 females and 3 males. The remaining cases were mostly purulent appendicitis. The true negative (normal appendix) for group I is, thus, 15 cases.

In group II; 48 patients the score was 7-9: 30 males, and 18 females: The average age was 25.5 years. Migratory pain and right iliac fossa tenderness were found in all patients, anorexia in $30 \%$ of patients, nausea and vomiting in all but one patient. The temperature was elevated in all but one patient; the average temperature was 38.1. Leukocytosis was evident in 95\% patients;

Table 1 Alvarado scoring system.

\begin{tabular}{|c|c|}
\hline Symptoms & Score \\
\hline Migratory right iliac fossa pain & 1 \\
\hline Nausea/ vomiting & 1 \\
\hline Anorexia & 1 \\
\hline Signs & 2 \\
\hline Rebound tenderness in right iliac fossa & 1 \\
\hline Elevated temperature & 1 \\
\hline Laboratory findings & 2 \\
\hline Leukocytosis & 1 \\
\hline Shift to the left of neutrophils & 10 \\
\hline Total
\end{tabular}

Table 2 Alvarado score distribution among patients.

\begin{tabular}{|c|c|}
\hline Alvarado Score & $\mathbf{n}$ \\
\hline $3-5$ & 2 \\
\hline $5-7$ & 50 \\
\hline $7-9$ & 48 \\
\hline Total & 100 \\
\hline
\end{tabular}

Table 3 Pathology types in 100 appendix specimens.

\begin{tabular}{|cc|}
\hline Type & Number of case \\
\hline Gangrenous & 30 \\
\hline Purulent & 37 \\
\hline Perforated & 4 \\
\hline Catarrhal & 9 \\
\hline Unremarkable & 20 \\
\hline
\end{tabular}

Table 4 Diagnostic accuracy of Alvarado score.

\begin{tabular}{|c|c|c|c|}
\hline Group & $n$ & $\begin{array}{c}\text { Confirmed } \\
\text { Appendicitis }\end{array}$ & Normal Appendix \\
\hline $\begin{array}{c}\text { I (Alvarado score } \\
\geq 7 \text { ) }\end{array}$ & 48 & 43 (True positive) & 5 ( False positive) \\
\hline $\begin{array}{c}\text { II (Alvarado score } \\
\leq 7)\end{array}$ & 52 & 37 (False negative) & 15 (True negative) \\
\hline
\end{tabular}

Sensitivity $54 \%$ Specificity $75 \%$ Positive predictive value $90 \%$ Negative predictive value $29 \%$ Diagnostic accuracy value $58 \%$

average 13,200 without left shift.The appendix was perforated in two male patients and unremarkable in five patients (the false positive), gangrenous in 29, and purulent in 12 .

The appendix was normal or unremarkable in 20 (14 females, 6 males) patients which mean a $20 \%$ negative appendectomy rate, and perforated in four (three males, one female) patients; perforation rate $4 \%$. The pathology results are shown in Table 3.

In this study, the sensitivity of Alvarado score was $54 \%$, specificity $75 \%$, positive predictive value $90 \%$, negative predictive value $29 \%$, and diagnostic accuracy value $58 \%$ as shown in Table 4.

\section{Discussion}

Although it is the most common cause of acute abdomen, acute appendicitis remains a challenging diagnosis because it is primarily a clinical diagnosis with a long list of differential diagnoses. The negative appendectomy rate in this series was $20 \%$ which is congruent with the rates reported in the literature of $8 \%$ to $33 \%$ [6]. Clinical scoring systems proved to be useful in the diagnosis of some surgical conditions. In the past few years deferent scoring systems developed to help diagnosis of acute apendicitis [7]. Although, many scoring systems advocated but most are sophisticated and difficult to implement in the real clinical situation [7]. On the other hand, Alvarado scoring system is a simple system that can be used easily in the clinic or emergency department [5]. To be helpful, the scoring system must be both sensitive and specific. The sensitivity in this study was $54 \%$ which is similar to that reported by Al Hashemy and Seleem [6] and lower than that reported by Lone et al of $88 \%$ [8]. The positive predicative value $90 \%$ in this study is close to that of other studies which may actually reflect the high prevalence of acute appendicitis $[9,10]$. Negative predictive value was $29 \%$, 
and diagnostic accuracy of 58\%. Our study shows that Alvarado scoring system is not sensitive enough to consider highly or routinely dependent on for the diagnosis of acute appendicitis. In addition, it may not be accurate in diagnosing acute appendicitis even in patients with score $\geq 7$ as shown in our series with 5 false positive cases out of 48 (10\%).

\section{References}

1 Addiss DG, Shaffer N, Fowler BS, Tauxe RV (1990) The epidemiology of appendicitis and appendectomy in the United States. Am J Epidemiol 132: 910-925.

2 Fitz RH (1886) Perforating inflammation of the vermiform appendix with special reference to its early diagnosis and treatment. Am J Med Sci (1827-1924, pp:321.

3 Dado G, Anania G, Baccarani U, Marcotti E, Donini A, et al. (2000) Application of a clinical score for the diagnosis of acute appendicitis in childhood: A retrospective analysis of 197 patients. J Pediatr Surg 35: $1320-1322$.

4 Fenyö G, Lindberg G, Blind P, Enochsson L, Oberg A, et al. (1997) Diagnostic decision support in suspected acute appendicitis: Validation of a simplified scoring system. Eur J Surg 163: 831-838.

\section{Conclusion}

In this study, Alvarado scoring system was found not sensitive in aiding diagnosis of acute appendicitis, probably due to the heterogeneous population studied. Further refinement and adjustments of the scoring system may be needed to improve sensitivity and decrease the controversy over its use routinely.

5 Alvarado A (1986) A practical score for the early diagnosis of acute appendicitis. Ann Emerg Med 15: 557-564.

6 Al Hashemy AM, Seleem MI (2004) Appraisal of the modified alvarado score for acute appendicits in adults. Saudi Med J 25: 1229-1231.

7 Ohmann C, Yang Q, Franke C (1995) Diagnostic scores for acute appendicitis. Abdominal Pain Study Group. Eur J Surg 161: 273-281.

8 Lone NA, Shah M, Wani KA, Peer GQ (2006) Modified Alvarado score in diagnosis of acute appendicitis. Ind J Pract Dr 3: 1-4.

9 Jan HI, Khan J (2007) Evaluation of modified Alvarado score in the diagnosis of acute appendicitis. Pak J Surg 23: 248-250.

10 Fenyo G (1987) Routine use of a scoring system for decision-making in suspected acute appendicitis in adults. Acta Chir Scand 153: 545551. 